\title{
Cross-Cultural Adaptation, Validity, and Reliability of the Persian Version of the Orebro Musculoskeletal Pain Screening Questionnaire
}

\author{
Asrin Shafeei ${ }^{1}$, Hamid Reza Mokhtarinia ${ }^{2}$, Azam Maleki-Ghahfarokhi ${ }^{3}$, Leila Piri $^{4}$ \\ ${ }^{1}$ Department of Occupational Therapy, Qods Hospital, Kurdistan University of Medical Sciences, Sanandaj, Iran \\ ${ }^{2}$ Department of Ergonomics, University of Social Welfare and Rehabilitation Sciences, Tehran, Iran \\ ${ }^{3}$ Student Research Committee, Faculty of Health, Tabriz University of Medical Sciences, Tabriz, Iran \\ ${ }^{4}$ Department of Ergonomics, University of Social Welfare and Rehabilitation Sciences, Tehran, Iran
}

\section{Study Design: Observational study.}

Purpose: To cross-culturally translate the Orebro Musculoskeletal Pain Screening Questionnaire (OMPQ) into Persian and then evaluate its psychometric properties (reliability, validity, ceiling, and flooring effects).

Overview of Literature: To the authors' knowledge, prior to this study there has been no validated instrument to screen the risk of chronicity in Persian-speaking patients with low back pain (LBP) in Iran. The OMPO was specifically developed as a self-administered screening tool for assessing the risk of LBP chronicity.

Methods: The forward-backward translation method was used for the translation and cross-cultural adaptation of the original questionnaire. In total, 202 patients with subacute LBP completed the OMPQ and the pain disability questionnaire (PDQ), which was used to assess convergent validity. 62 patients completed the OMPQ a week later as a retest.

Results: Slight changes were made to the OMPO during the translation/cultural adaptation process; face validity of the Persian version was obtained. The Persian OMPQ showed excellent test-retest reliability (intraclass correlation coefficient $=0.89$ ). Its internal consistency was 0.71 , and its convergent validity was confirmed by good correlation coefficient between the OMPO and PDQ total scores $(r=0.72, p<0.05)$. No ceiling or floor effects were observed.

Conclusions: The Persian version of the OMPQ is acceptable for the target society in terms of face validity, construct validity, reliability, and consistency. It is therefore considered a useful instrument for screening Iranian patients with LBP.

Keywords: Disability; Orebro Musculoskeletal Pain Screening Questionnaire; Persian version; Reliability; Validity

\section{Introduction}

Work-related musculoskeletal disorders (MSDs) constitute a huge global health problem, leading to substantial economic and human costs as well as having a negative impact on the quality of life. The most frequently reported subcategory of MSDs is injuries to the back and spine, accounting for $51.7 \%$ of cases [1]. Low back pain (LBP) is a very common health problem worldwide and a major cause of disability, affecting performance and general wellbeing. It is considered to be among the top 10 causes of employee absenteeism in the workplace and accounts

Received Dec 5, 2016; Revised Jan 5, 2017; Accepted Jan 16, 2017

Corresponding author: Hamid Reza Mokhtarinia

University of Social Welfare and Rehabilitation Sciences, Kodakyar Ave., Daneshjo Blvd., Evin, PC: 1985713834, Tehran, Iran

Tel: +98-21-22180119, Fax: +98-21-22180119, E-mail: hrmokhtarinia@yahoo.com 
for about $12.5 \%$ of all sick leaves worldwide [2]. Mousavi et al. [3] reported LBP to be common in Iran and an important cause of disease burden, in particular among the most productive ages (15 to 69 years) in both males and females. They reported the prevalence of LBP in the Iranian general population, working population, school children, and pregnant women was in the range of $14.4 \%-$ 84.1\% [3].

The transition from acute back pain to chronic disability is one of the major concerns in the management of LBP, given its high social and financial costs [4]. Recent studies have revealed psychosocial factors that are related to future disability and play an important role in the development of chronicity and delayed return to work; these are referred to as "yellow flags" [5]. The early and proper detection of LBP patients at risk of disability and the application of effective rehabilitation strategies are important not only for patients and health care professionals but also for governments and policy makers for developing early interventions with the aim of minimizing work absenteeism and health care costs as well as enhancing the quality of life [6].

Several self-reported back-specific questionnaires have been developed for individuals with LBP [7-9]. The Orebro Musculoskeletal Pain Screening Questionnaire (OMPQ), developed by Linton and Hallden [10] in 1998, is used in various clinical settings $[5,11]$. One specific advantage of the OMPQ is assisting in the early detection of yellow flags [12]. It is considered a reference measure with regard to screening subacute LBP patients at risk of disability and persistent pain [11]. This instrument has been validated across multiple clinical settings and cultures [13-15]. The use of an instrument in a different culture requires that the items are translated well linguistically and that they are adapted culturally to maintain the content validity of the tool at the same conceptual level across different cultures [16]. In Iran, prior to this study, there has been no specific evaluation measure for screening LBP patients at the risk of disability. Therefore, the purpose of this study was the translation and cultural adaptation of the OMPQ for use in Iran and the quantitative evaluation of the validity and reliability of its Persian version.

\section{Materials and Methods}

The original OMPQ in English [10] was used as the basis for cross-cultural adaptation in this study. The Persian version of pain disability questionnaire (PDQ) [17] was applied to test the concurrent validity of the questionnaire.

\section{Translation and cross-cultural adaptation}

The procedure adopted in this study was mainly based on the protocol of Bullinger et al. [18]. There are two major steps when translating any questionnaire from its original language into another language: cultural adaptation and evaluation of the validity and reliability of the questionnaire. Permission for translation was obtained from the initial developer (Dr. S.J. Linton, Orebro University, Sweden). During forward translation, two independent native Persian speakers translated the OMPQ from English to Persian. The translators and researchers then compared the two translated versions with the original questionnaire [18] and reached a consensus. Following this, two independent native English speakers who were proficient in the Persian language independently performed a backward translation, and after consultation, they made the required revisions. The resulting translated English version was compared with the original one with respect to conceptual equivalence by a team of translators and researchers (a physiotherapist, a psychologist, and an occupational medicine specialist) [18]. This pre-final Persian OMPQ questionnaire was then piloted in the presence of one of the assessors (A.S.) on 30 patients with LBP who volunteered to participate in the study and provided written informed consent. These participants were not included in the main study. The simplicity, clarity, and general translation quality of the questionnaire were verified by most pilot study participants. After iteratively implementing points raised in the patients' feedback, the final version was sent to Dr. Linton, who confirmed the conceptual equality of the created backward English questionnaire and the original OMPQ.

\section{Participants}

A sample of 202 native Persian speakers with subacute LBP was recruited as volunteers from rehabilitation centers in Tehran, Iran, between April 2014 and December 2014. The inclusion criteria were age between 22 and 52 years and subacute LBP of less than 10-week duration. Exclusion criteria were pregnancy, recent surgery, neurological impingement syndrome, tumors, suspected inflammatory 
arthritis, fractures, or requirement of referral to a further medical specialist. The participants were fully informed about the study and signed an informed consent form. The study was approved by the ethics committee of the University of Social Welfare and Rehabilitation Sciences.

In the first session, the participants completed both the OMPQ and PDQ in the clinic waiting room. To evaluate test-retest reliability, 62 participants with the assumption of clinical stability completed the OMPQ at the same location 3 to 7 days later. The time interval between test and retest was selected to reduce the possibility of the participants remembering their previous responses [19].

\section{Instruments}

The OMPQ is a 25 -item self-report questionnaire for the early diagnosis of patients susceptible to the progression of permanent and long-term musculoskeletal pain $[10,12]$. It is valuable in predicting disability caused by occupational disorders and in predicting delays in returning to work because of musculoskeletal problems [20]. In addition, this screening tool enables a practitioner to identify possible risk factors and apply appropriate interventions to reduce the risk of long-term disability in injured workers [21]. The OMPQ can be completed 5 to 10 minutes; the score (in a range of 0-210) is derived from the sum of the scores of 21 items, with higher scores indicating a greater risk of chronic disability [21]. The predictability, validity, and reliability of the OMPQ have been evaluated in countries such as Sweden [21] and France [20].

The PDQ, developed by Anagnostis et al. [17] in 2004, is used to measure pain caused by work [18]. It has two parts, covering functional and social-mental conditions [17]. The score (in a range of 0 to 150 , indicating optimal function to complete disability) is calculated as the sum of the scores of 15 items. The PDQ has been translated into various languages, and its validity and reliability have been demonstrated [17]. The Persian version of the PDQ was created by Marbouti et al. [22] in 2011, and its psychometric properties have been reported. In this study, the Persian PDQ was used as an external measure of disability.

\section{Evaluation of psychometric characteristics}

Psychometric characteristics include test-retest reliability, internal consistency, construct validity, face validity, and ceiling and floor effects. In this study, we assessed two aspects of reliability as test-retest reliability and internal consistency. Intraclass correlation coefficient (ICC 2.1) was used to evaluate the relative reliability. ICC $\geq$ 0.70 was considered acceptable for test-retest reliability [23]. A paired $t$-test was applied to the results of two sessions with the aim of systematically validating the mean OMPQ score. To estimate the measurement accuracy, the standard error of measurement (SEM) was obtained as a reliability index using a variance table [23]. Internal consistency was assessed by Cronbach's a coefficient for the whole questionnaire; acceptable values were those at or above 0.70 [24]. The face validity was determined, as described above, by recruiting 30 patients with LBP to a pilot study to assess whether the items in the questionnaire were simple, clear, and understandable [25]. To measure the convergent validity of the OMPQ, the participants in the main study completed the PDQ at the same time, with the expectation that those with higher scores in the OMPQ would have higher scores in the PDQ. The correlation between the two questionnaires was evaluated by Spearman correlation analysis, with no assumption that the data were normally distributed. Ceiling and floor effects were assessed by counting the number of participants who scored the minimum (0) or maximum (210) OMPQ score in the first session, taking the effects into consideration if $15 \%$ of the participants scored either of these values [26]. All statistical analyses were conducted using the SPSS ver. 19.0 (IBM Corp., Armonk, NY, USA) for Windows. The significance level was set at $p<0.05$.

\section{Results}

\section{Participant characteristics}

In total, 202 patients suffering from LBP (54 men, 148 women) participated in this study. The mean age was 34.38 (standard deviation [SD], 6.18) years, mean total work experience was 8.85 (SD, 5.41) years, and mean work hours per week were 38.11 (SD, 6.06) hours. The mean duration of their condition was 50.60 (SD, 13.70) days. The participants' demographic and occupational characteristics are presented in Table 1.

\section{Translation process and cultural adaptation}

The cultural adaptation process included forward translation, assessment of the quality of the translation, and 
Table 1. Occupational and demographic characteristics of participants completing the OMPQ $(n=202)$

\begin{tabular}{|c|c|c|}
\hline Variable & No. $(\%)$ & Mean \pm SD \\
\hline \multicolumn{3}{|l|}{ Sex } \\
\hline Male & $54(26.7)$ & - \\
\hline Female & $148(73.3)$ & - \\
\hline \multicolumn{3}{|l|}{ Educational level } \\
\hline Less than high school diploma & $3(1.5)$ & - \\
\hline High school diploma & $53(26.2)$ & - \\
\hline BSc and higher & $146(72.3)$ & - \\
\hline \multicolumn{3}{|l|}{ Marital status } \\
\hline Single & $36(17.8)$ & \\
\hline Married & 166 (82.2) & \\
\hline Age (yr) & - & $34.4 \pm 6.2$ \\
\hline Duration of disease (day) & - & $50.6 \pm 13.7$ \\
\hline Work experience (yr) & - & $8.9 \pm 5.4$ \\
\hline Working hours per week & - & $38.1 \pm 6.1$ \\
\hline
\end{tabular}

OMPQ, Orebro Musculoskeletal Pain Screening Questionnaire; SD, standard deviation; BSc, Bachelor of Science.

backward translation. During the forward-backward translation process, it was decided to change the first four demographic items into an interrogative form, transforming the 25 -item original questionnaire into a 21 -item instrument. In the demographics section, questions related to marital status, work experience, average working hours/week, and education level were added, whereas the question "Are you born in Australia?" was removed. Six participants proposed changing the items numbered 6, 17, and 18 and seven participants proposed adding a guide to the questionnaire. Item 6 "Show your mean pain during three last months with one of following numbers" was changed to "How severe was your pain during the past 3 months on a scale of $1-10$ ?" and items 17 and 18 were changed from "I can do light work up to one hour" and "I can walk up to one hour" to "I can do light work for 1 hour" and "I can walk for 1 hour," respectively. Additional information was introduced for guidance at the beginning of the questionnaire: "In some questions, there is a scale of 1-10, which shows minimum and maximum scores. Please circle the number that correctly indicates your current condition." The final Persian OMPQ is presented in Appendix 1.

\section{Evaluation of psychometric characteristics}

As shown in Table 2, ICC for the whole questionnaire was 0.89 , indicative of very high and optimum reliability. Paired $t$ test results showed that there was no significant difference between scores for the OMPQ in the two sessions, which indicated that there was no systematic error $(p=0.432)$. Cronbach's $\alpha$ value for the whole questionnaire was 0.71; when each item was omitted individually, Cronbach's $\alpha$ values were in the range of $0.66-0.76$. Based on these statistical results, the contribution of questions 1,13 , and 15 was lower than the others; if omitted, Cronbach's a value would increase to 0.76 . SEM for the questionnaire was 3.2, indicating absolute reliability (Table 2).

The convergent validity of the OMPQ and PDQ was assessed using Spearman's correlation analysis. The results showed a highly significant correlation between the total scores for the Persian OMPQ and PDQ $(r=0.72, p<0.05)$, demonstrating the convergent validity of the OMPQ. The percentages of the respondents who obtained the minimum score $(63,1.5 \%)$ or maximum score $(155,0.5 \%)$ for the OMPQ did not reach the threshold of 15 ; therefore, ceiling and floor effects did not need to be taken into consideration. The minimum and maximum scores for the PDQ were found to be 12 and 130, respectively.

\section{Discussion}

The purpose of this study was the translation and cultural adaptation of the OMPQ questionnaire into Persian and 
Table 2. Test-retest reliability and internal consistency of the Persian version of OMPO

\begin{tabular}{|c|c|c|c|c|c|c|}
\hline \multirow{2}{*}{ Questionnaire } & \multicolumn{2}{|c|}{ Mean \pm SD } & \multirow{2}{*}{ SEM } & \multirow{2}{*}{ ICC } & \multirow{2}{*}{$\begin{array}{l}95 \% \text { Confidence } \\
\text { interval of ICC }\end{array}$} & \multirow{2}{*}{$\begin{array}{l}\text { Cronbach's } \\
\text { alpha }(n=62)\end{array}$} \\
\hline & Test ( $n=62)$ & Retest ( $n=62$ ) & & & & \\
\hline Persian version of OMPO & $104.7 \pm 7.66$ & $105.2 \pm 7.122$ & 3.2 & 0.89 & $0.70-0.93$ & 0.71 \\
\hline
\end{tabular}

Values for the test and retest are presented as mean $\pm S D$.

Cronbach's alpha is based on the first assessment day.

OMPQ, Orebro Musculoskeletal Pain Screening Questionnaire; SD, standard deviation; SEM, standard error of measurement; ICC, intraclass correlation coefficient.

measurement and evaluation of the psychometric characteristics of this instrument.

In the original questionnaire, the early questions about demographic information did not contribute to the total score; during the process of cultural adaptation, the team of translators and experts agreed to present these in a separate section at the beginning of the questionnaire. Additional items about marital status, work experience, mean work hours/week, and educational level were included in the questionnaire. The question "Are you born in Australia?" was removed without replacement because the majority of people in Iran are Iranian. To enhance clarity, a brief explanation that " 0 indicates minimum and 10 indicates maximum" was added at the start of the questionnaire.

Item 6 "Show your mean pain during three last months" was changed to "How severe was your pain during the past 3 months?" because pain severity is more tangible in Persian people; and, as described earlier, "up to" in items 17 and 18 was changed to "for." Again, the reason for these changes was to improve clarity and enhance the ease of understanding based on the feedback provided during the pilot study. However, in general, the participants had no difficulty in understanding and completing the Persian version of the OMPQ.

The results demonstrated high agreement between measurements recorded on two occasions a week apart. The test-retest reliability analysis results showed ICC $=0.89$, indicating high reliability [23]. Linton and Hallden [10], in a pilot study for the original OMPQ that included 27 participants and a test-retest interval of one week, obtained a Pearson correlation coefficient of 0.83 . Thus, the result for the test-retest reliability of the Persian OMPQ was similar to that for the original OMPQ. It was also similar to the results of Opsommer et al. [27] (ICC=0.89) and Grotle et al. [25] (ICC=0.9).

SEM obtained in this study (3.2) was much lower than that obtained for the Swiss population in the study of Opsommer et al. [27] (10.1), indicating that the absolute reliability of the Persian OMPQ was higher. This is important because the magnitude of the change in the OMPQ questionnaire should be greater than SEM when evaluating real changes over time.

The assessment of the internal consistency of the items showed homogeneity between individual participants' total scores for one sample in the two tests performed a week apart. Cronbach's $a$ value for the Persian OMPQ was 0.71; when items were extracted individually, the value varied between 0.69 and 0.76 . The results should be interpreted with caution because Cronbach's a value depends on the number of items in a scale or questionnaire, item interrelatedness, and dimensionality [28]. The result for internal consistency obtained in this study was close to Cronbach's a value achieved for the Brazilian-Portuguese version of the OMPQ [29] but not similar to that achieved for the Norwegian version [25].

Convergent validity was assessed by evaluating the correlation between the total scores of the equivalent Orebro questionnaire and PDQ. The results showed a significant correlation between the total scores for the PDQ and OMPQ ( $r=0.72, p<0.05)$; however, this significant correlation was expected given that the PDQ also includes questions regarding pain and disability. In comparison, Nonclercq and Berquin [20] found a moderate correlation between the Oswestry disability index (ODI) and OMPQ scores. One explanation for this difference was the smaller sample size ( $\mathrm{n}=91)$ in that study. In addition, they used a nonvalidated French version of the ODI [20].

As expected, no ceiling or floor effects were observed in the present study. This was in accordance with the result for the Brazilian-Portuguese version [29] and indicated the capability of the Persian version of the OMPQ to identify clinical differences. One of the strengths of this study was the synchronous evaluation of the question- 
naire's validity and reliability along with adaptation in a particular culture/language. A 1-week time interval was used for the test-retest reliability study; a different interval may have resulted in a different outcome. For phenomena that change over time, shorter time intervals should be selected. However, short intervals may lead to an artificial estimation of reliability because participants may regulate their answers by remembering previous answers or through training effects [27].

One of the limitations of this study was that the predictability of the Persian OMPQ was not assessed; such an assessment is strongly recommended in future investigations. Several other questionnaires could have been selected to assess convergent validity; however, similar results would be expected. Further studies with larger sample sizes are needed to examine clinical application and the disability predictive capabilities of the questionnaire.

\section{Conclusions}

This study established the internal consistency, construct validity, and reliability of the Persian OMPQ in a population of patients suffering from subacute LBP $(n=202)$. The results of this study provide further support for the standard use of OMPQ in primary care settings.

\section{Conflict of Interest}

No potential conflict of interest relevant to this article was reported.

\section{Acknowledgments}

The authors appreciate Dr. S.J. Linton for permission of translating the OMPQ into Persian. They also like to acknowledge kind assistance of the participants. This paper was extracted from a thesis by A. Shafeei written to fulfill requirements for master degree in ergonomics, University of Social Welfare and Rehabilitation Sciences, Tehran, Iran.

\section{ORCID}

Asrin Shafeei: https://orcid.org/0000-0003-2892-2229

Hamid Reza Mokhtarinia: https://orcid.org/0000-0002-5181-4894

Azam Maleki-Ghahfarokhi: https://orcid.org/0000-0001-8222-2659

Leila Piri: https://orcid.org/0000-0003-3347-2859

\section{References}

1. Praemer A, Furner S, Rice DP. Musculoskeletal conditions in the United States. Park Ridge: American Academy of Orthopaedic Surgeons; 1992. p.1-99.

2. Vos T, Flaxman AD, Naghavi M, et al. Years lived with disability (YLDs) for 1160 sequelae of 289 diseases and injuries 1990-2010: a systematic analysis for the Global Burden of Disease Study 2010. Lancet 2012;380:2163-96.

3. Mousavi SJ, Akbari ME, Mehdian H, et al. Low back pain in Iran: a growing need to adapt and implement evidence-based practice in developing countries. Spine (Phila Pa 1976) 2011;36:E638-46.

4. Kovacs FM, Abraira V, Zamora J, Fernandez C; Spanish Back Pain Research Network. The transition from acute to subacute and chronic low back pain: a study based on determinants of quality of life and prediction of chronic disability. Spine (Phila Pa 1976) 2005;30:1786-92.

5. Heneweer H, van Woudenberg NJ, van Genderen F, Vanhees L, Wittink H. Measuring psychosocial variables in patients with (sub) acute low back pain complaints, at risk for chronicity: a validation study of the Acute Low Back Pain Screening QuestionnaireDutch Language Version. Spine (Phila Pa 1976) 2010; 35:447-52.

6. Boersma K, Linton SJ. Screening to identify patients at risk: profiles of psychological risk factors for early intervention. Clin J Pain 2005;21:38-43.

7. Beurskens AJ, de Vet HC, Koke AJ, van der Heijden GJ, Knipschild PG. Measuring the functional status of patients with low back pain: assessment of the quality of four disease-specific questionnaires. Spine (Phila Pa 1976) 1995;20:1017-28.

8. Linton SJ. Early identification and intervention in the prevention of musculoskeletal pain. Am J Ind Med 2002;41:433-42.

9. Hill JC, Dunn KM, Main CJ, Hay EM. Subgrouping low back pain: a comparison of the STarT Back Tool with the Orebro Musculoskeletal Pain Screening Questionnaire. Eur J Pain 2010;14:83-9.

10. Linton SJ, Hallden K. Can we screen for problematic back pain? A screening questionnaire for predicting outcome in acute and subacute back pain. Clin J Pain 1998;14:209-15.

11. Dunstan DA, Covic T, Tyson GA, Lennie IG. Does 
the Orebro Musculoskeletal Pain Questionnaire predict outcomes following a work-related compensable injury? Int J Rehabil Res 2005;28:369-70.

12. Linton SJ, Nicholas M, MacDonald S. Development of a short form of the Orebro Musculoskeletal Pain Screening Questionnaire. Spine (Phila Pa 1976) 2011; 36:1891-5.

13. Sivan M, Sell B, Sell P. A comparison of functional assessment instruments and work status in chronic back pain. Eur J Phys Rehabil Med 2009;45:31-6.

14. Gabel CP, Burkett B, Neller A, Yelland M. Can longterm impairment in general practitioner whiplash patients be predicted using screening and patientreported outcomes? Int J Rehabil Res 2008;31:79-80.

15. Hockings RL, McAuley JH, Maher CG. A systematic review of the predictive ability of the Orebro Musculoskeletal Pain Questionnaire. Spine (Phila Pa 1976) 2008;33:E494-500.

16. Herdman M, Fox-Rushby J, Badia X. 'Equivalence' and the translation and adaptation of health-related quality of life questionnaires. Qual Life Res 1997;6: 237-47.

17. Anagnostis C, Gatchel RJ, Mayer TG. The pain disability questionnaire: a new psychometrically sound measure for chronic musculoskeletal disorders. Spine (Phila Pa 1976) 2004;29:2290-302.

18. Bullinger M, Alonso J, Apolone G, et al. Translating health status questionnaires and evaluating their quality: the IQOLA Project approach. International Quality of Life Assessment. J Clin Epidemiol 1998;51: 913-23.

19. Bennell K, Bartam S, Crossley K, Green S. Outcome measures in patellofemoral pain syndrome: test retest reliability and inter-relationships. Phys Ther Sport 2000;1:32-41.

20. Nonclercq O, Berquin A. Predicting chronicity in acute back pain: validation of a French translation of the Orebro Musculoskeletal Pain Screening Ques- tionnaire. Ann Phys Rehabil Med 2012;55:263-78.

21. Linton SJ, Boersma K. Early identification of patients at risk of developing a persistent back problem: the predictive validity of the Orebro Musculoskeletal Pain Questionnaire. Clin J Pain 2003;19:80-6.

22. Marbouti L, Jafari H, Nourizadeh-Dehkordi S, Behtash H. Pain-related disability measurement: the cultural adaptation and validation of "pain disability index (PDI)" and "pain disability questionnaire (PDQ)" among Iranian low back pain patient. Med J Islam Repub Iran 2011;25:27-34.

23. Atkinson G, Nevill AM. Statistical methods for assessing measurement error (reliability) in variables relevant to sports medicine. Sports Med 1998;26:21738.

24. Fayers PM, Machin D. Quality of life: the assessment, analysis and interpretation of patient-reported outcomes. Chichester: John Wiley \& Sons; 2000.

25. Grotle M, Vollestad NK, Brox JI. Screening for yellow flags in first-time acute low back pain: reliability and validity of a Norwegian version of the Acute Low Back Pain Screening Questionnaire. Clin J Pain 2006; 22:458-67.

26. McHorney CA, Tarlov AR. Individual-patient monitoring in clinical practice: are available health status surveys adequate? Qual Life Res 1995;4:293-307.

27. Opsommer E, Hilfiker R, Raval-Roland B, Crombez G, Rivier G. Test-retest reliability of the Orebro Musculoskeletal Pain Screening Questionnaire and the Situational Pain Scale in patients with chronic low back pain. Swiss Med Wkly 2013;143:w13903.

28. Shevlin M, Miles JN, Davies MN, Walker S. Coefficient alpha: a useful indicator of reliability? Pers Individ Dif 2000;28:229-37.

29. Fagundes FR, Costa LO, Fuhro FF, Manzoni AC, de Oliveira NT, Cabral CM. Orebro Questionnaire: short and long forms of the Brazilian-Portuguese version. Qual Life Res 2015;24:2777-88. 
Appendix 1. Orebro Musculoskeletal Pain Questionnaire

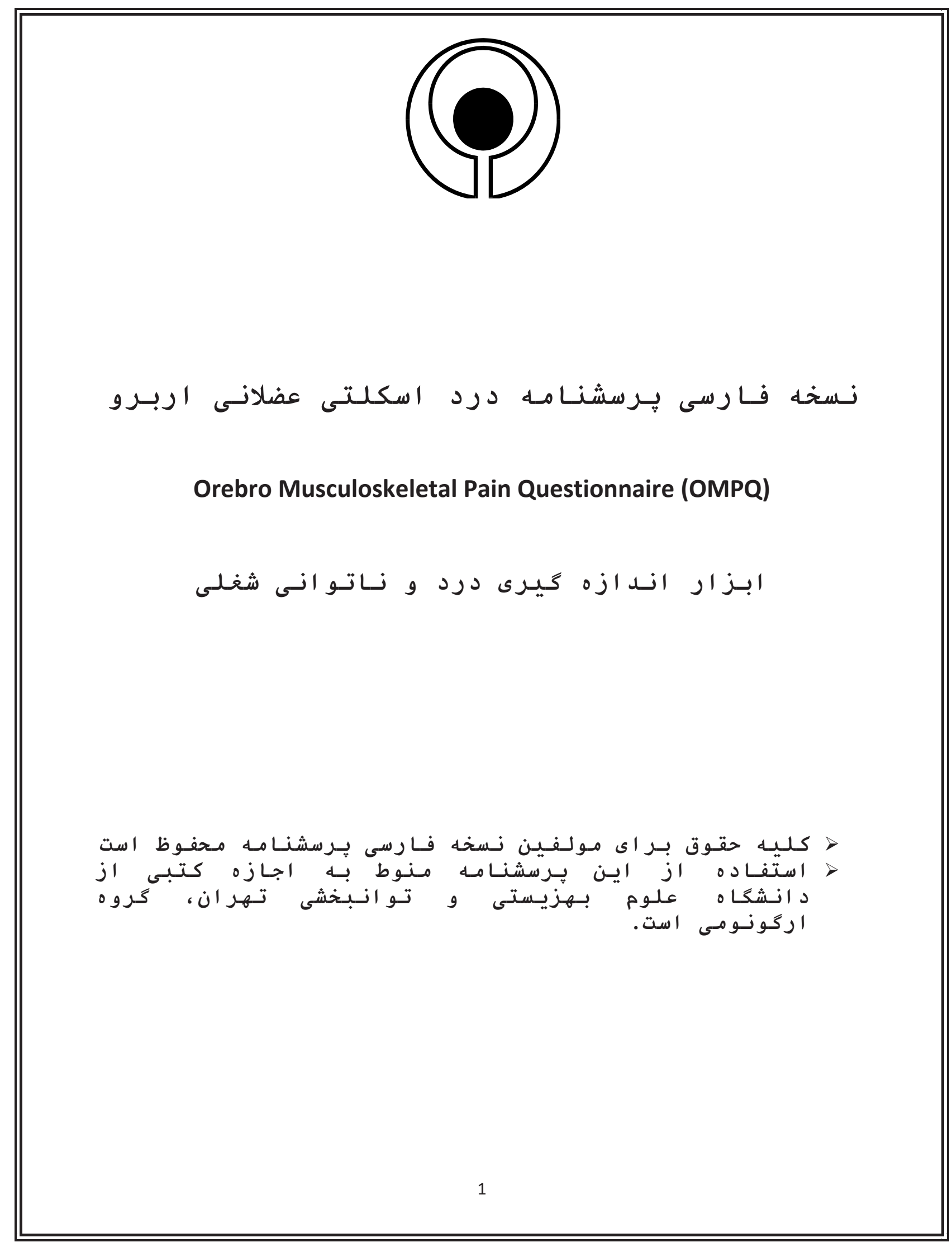




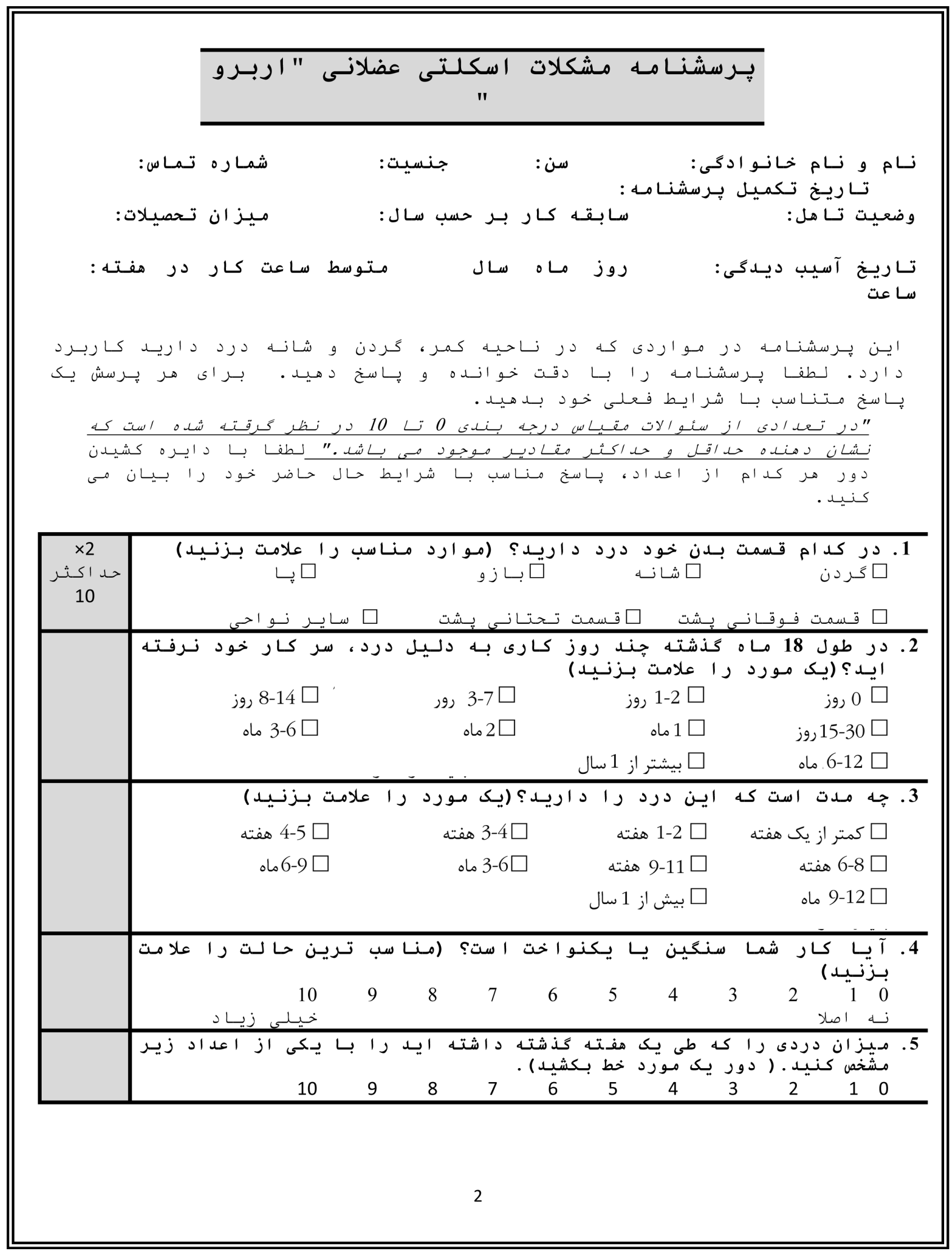




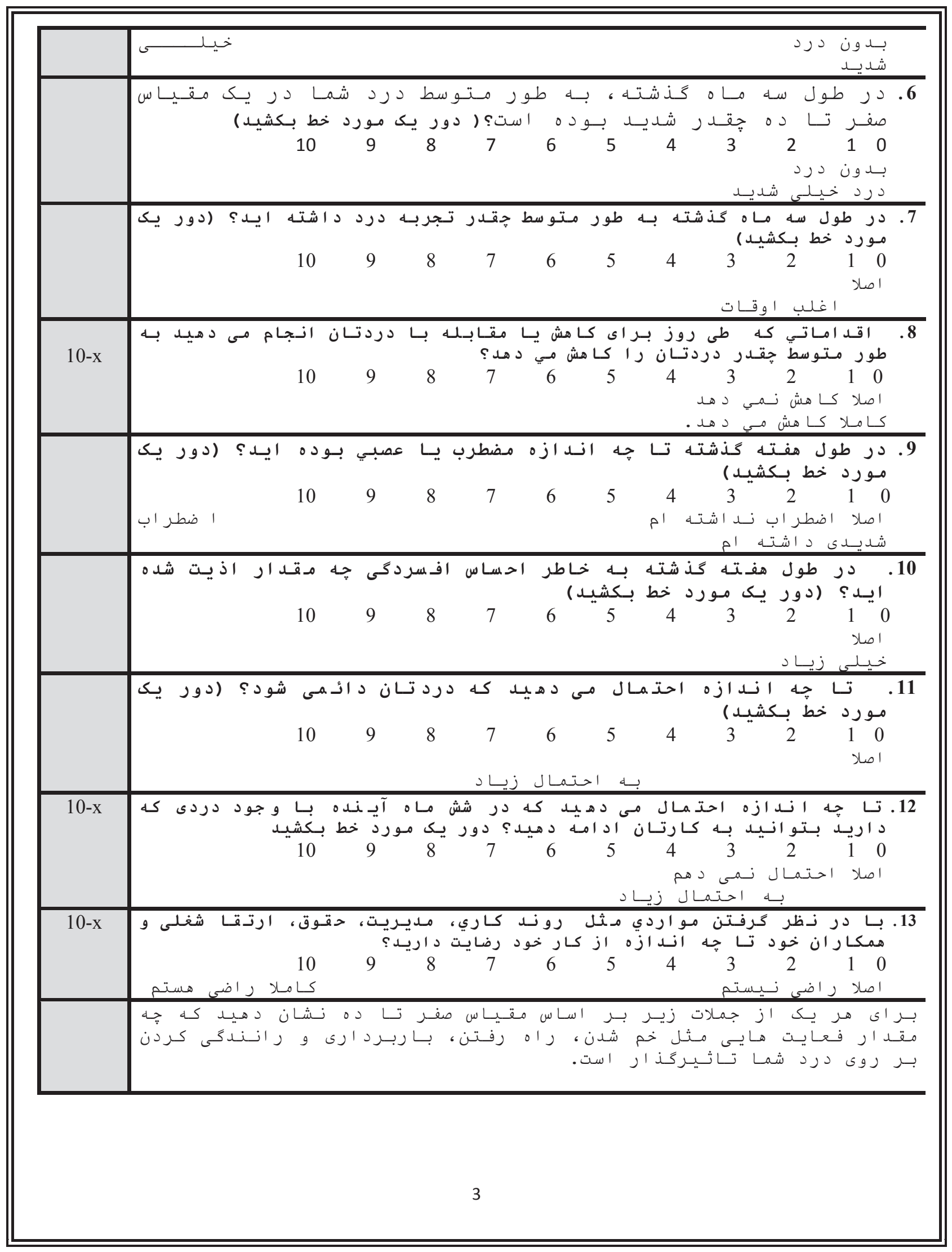




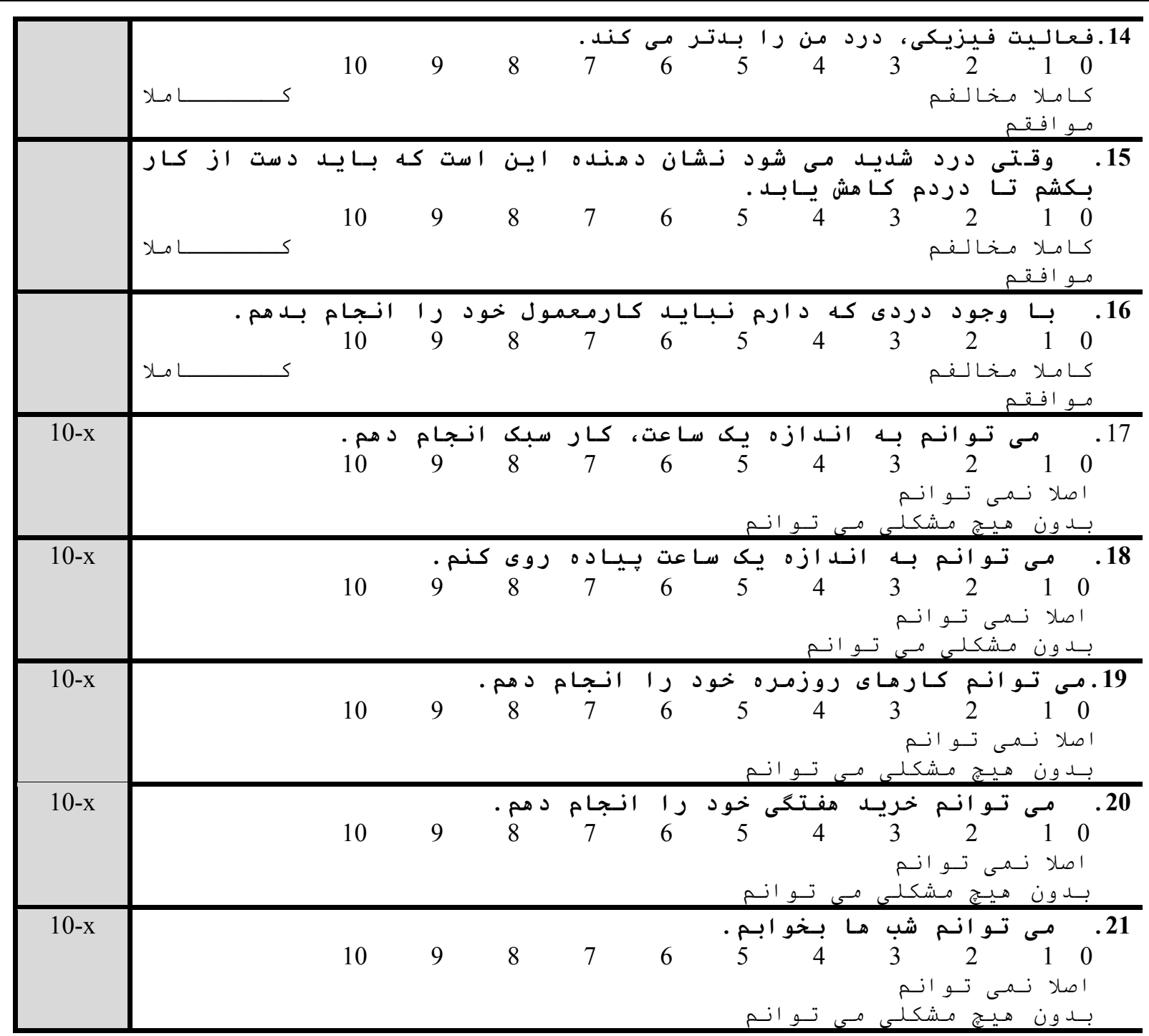

